\title{
Modification of Polymer Substrates with Extreme Ultraviolet Potential Application in Cancer Cell Identification
}

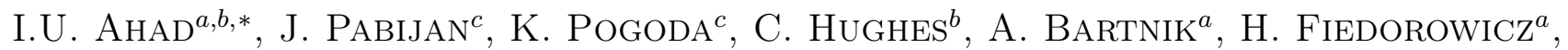 \\ M. LEKKA ${ }^{c}$ AND D. BRABAZON ${ }^{b}$ \\ ${ }^{a}$ Institute of Optoelectronics, Military University of Technology, 00-908 Warsaw, Poland \\ ${ }^{b}$ Advanced Processing Technology Research Centre, School of Mechanical and Manufacturing Engineering, \\ Faculty of Engineering and Computing, Dublin City University, Dublin 9, Ireland \\ ${ }^{c}$ Institute of Nuclear Physics, Polish Academy of Sciences, \\ E. Radzikowskiego 152, 31-342 Krakow, Poland
}

\begin{abstract}
During the last two decades, the development of laboratory scale extreme ultraviolet sources has been intensified due to growing interest in use of extreme ultraviolet photons for various applications in science and technology. In this study, we present a potential application of extreme ultraviolet sources for surface modification of polymers to be used as substrates for cancer cell identification. The surface modification of polytetrafluoroethylene (PTFE) polymer samples was performed by a lab scale compact laser-plasma extreme ultraviolet source based on a double-stream gas-puff target. The gas target was irradiated with a $3 \mathrm{~ns} / 0.8 \mathrm{~J} \mathrm{Nd}$ :YAG laser pulse at $10 \mathrm{~Hz}$. Reference HCV29 non-malignant transitional epithelium and T24 bladder cancer cells adhesion and proliferation studies on pure and extreme ultraviolet sources modified PTFE surfaces were performed. The extreme ultraviolet modified surfaces demonstrated regular increase in cancer cell proliferation comparing to pristine sample. Initial results indicate that extreme ultraviolet treated substrates can facilitate the identification of cancer cells.
\end{abstract}

DOI: 10.12693/APhysPolA.133.283

PACS/topics: 81.05.Lg, 81.40.Wx, 81.16.Rf, 81.65.-b

\section{Introduction}

The interest in miniaturizing of laboratories to a smallscale device, lab on a chip (LOC), for biological testing is growing at fast pace. However, material selection for LOC substrates for particular biological applications is still a challenge due to indiscriminate biological models $[1,2]$. The foremost important characteristic required by any substrate is strong cell adhesion. The process of cell adhesion to substrates can be modulated by surface chemistry, morphological structure, and mechanical properties of substrate materials [3]. The physical and chemical properties of a substrate influence the cell attachment, adhesion and spreading. Particularly, the surface topography, surface roughness and surface free energy could play important role in providing focal adhesion sites for cell attachment. Moreover, the mechanism of cell attachment varies with different cell types and surface properties. A number of materials have been investigated and used as substrates for LOCs [4-8]. However, surface treatment and modification is often required to make the material suitable for particular application. A number of surface treatment techniques were used and currently being developed. However, these techniques often require multi-stage treatments, long processing time and produce undesirable effects [2]. In this study, a potential application of extreme ultraviolet (EUV) surface structuring of

*corresponding author; e-mail: inamul.ahad@dcu.ie polymers to be used as substrates for cancer cell investigations is presented. Polytetrafluoroethylene (PTFE) polymer films surfaces were modified using laser plasma EUV source under different conditions. The cell culture studies on pure and EUV modified PTFE surfaces were performed using reference HCV29 non-malignant transitional epithelium and T24 bladder cancer cells. The EUV modified surfaces demonstrated strong cell adhesion and systematic proliferation over four days of cell culture studies.

\section{Materials and methods}

PTFE foils $0.1 \mathrm{~mm}$ thick obtained from Goodfellow Cambridge Limited, UK were cut into $12 \mathrm{~mm}$ by $12 \mathrm{~mm}$ size, and mounted on an $X Y Z$ translation stage in the interaction chamber of the EUV source. The detailed description of the source is given elsewhere [9-11]. The polymer sample holder was kept $2 \mathrm{~mm}$ away from the EUV collector focal point. The translationary movements along $X$ and $Y$ planes allow irradiation of 10 by $10 \mathrm{~mm}$ area of the polymer samples. The polymer samples were irradiated with 50 or $300 \mathrm{EUV}$ shots at $10 \mathrm{~Hz}$ repetition rate in the presence of EUV-ionized nitrogen gas in the interaction region [12]. The detailed arrangement and working of the source on PTFE and other polymers for application in biomedical engineering are documented before $[11,13]$. Cells grown on samples for 24, 48,72 , and $96 \mathrm{~h}$ and prepared for fluorescent microscopy according to procedures described elsewhere [14]. The fluorescent images of the samples were obtained using 
commercially available optical microscope Olympus XI71 equipped with a X-Cite Series 120Q Lumen Dynamics lamp.

\section{Results and discussion}

The cell culture studies based on direct cell material contact were performed in order to evaluate the cell attachment and adhesion on pristine and EUV modified PTFE samples irradiated for $5 \mathrm{~s}$ and $33.3 \mathrm{~s}$ per $\mathrm{mm}$ along horizontal direction (hereinafter referred to 50 shots and 300 EUV shots samples, respectively). The EUV processing resulted in formation of regular and pronounced wall type structures and modification of the surface chemical properties as described previously [13].

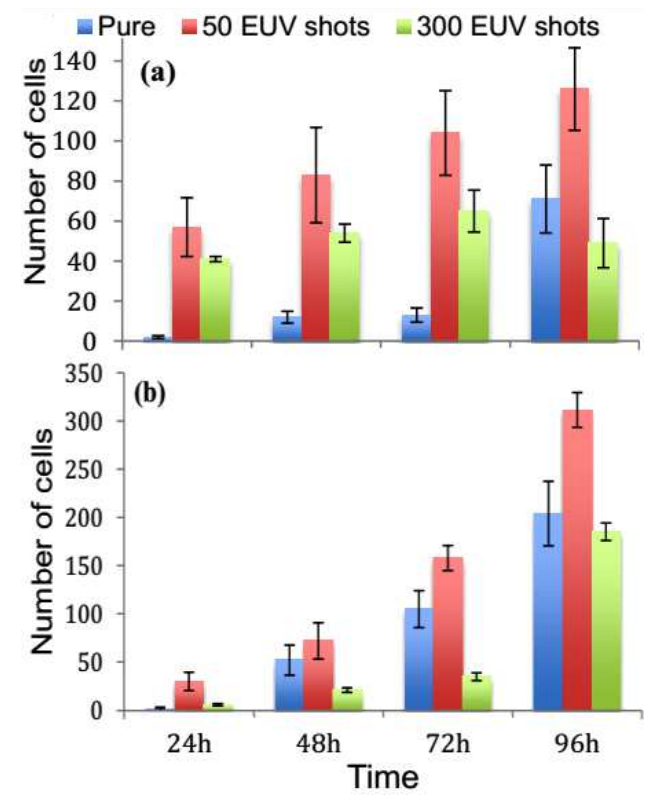

Fig. 1. (a) Average number of reference HCV29 cells attached to pure and EUV modified surfaces during four-day cell culture, (b) average number of T24 cancer cells attached to pure and EUV modified surfaces during four-day cell culture.

The HCV29 and T24 average cell number attached to polymer surface over the four day cell culture are summarized in graphical representation in Fig. 1a and b, respectively. The pure PTFE surface displayed no or weak HCV29 cell attachment on the first three days and moderate attachment on the fourth day. In case of 50 shots sample, the HCV29 cells demonstrated strong cell adhesion and good proliferation from first day and continue to grow over next 3 days. In case of PTFE surfaces irradiated with 300 EUV shots, weak HCV29 cell adhesion and proliferation were observed. The average number of cells attached to surface increased for first three days and decreased on fourth day (Fig. 1). The cells were mostly in elongated in shape and dispersed, however, some clusters of cells were also observed in case of 300 shots sample. In case of cancerous T24 cell, the cells start to attach after $48 \mathrm{~h}$ on pure PTFE sample and continue to grow for next two days. In case of 50 shots sample, T24 cells demonstrated similar cell attachment and proliferation behavior as of reference HCV29, however, more in number. The results of cancerous T24 cell culture on PTFE surfaces irradiated with 300 EUV pulses demonstrated very weak cell adhesion for first three days of cell culture. Unlike HCV29, the cells were mostly attached to the substrate in clusters, however single dispersed cells could also be observed. Earlier studies showed that EUV processing of PTFE samples gradually oxidise the polymer surface [13]. PTFE sample irradiated with 50 EUV shots demonstrated best cancer cell adhesion and proliferation results (Fig. 1a,b). Therefore, combining results from both studies suggests that cancer cells preferentially grow on less oxidized and wall type patterned surfaces with higher surface roughness.

\section{Conclusion}

The cancer cells with different metastatic potential could be identified on the basis of pleomorphic and mechanical phenotype. Extreme ultraviolet surface modification has been introduced to produce structured substrates appropriate for cancer cells identification. The EUV irradiated PTFE samples were successfully able to differentiate between non-malignant transitional epithelium HCV29 reference cells and malignant T24 bladder cancer cells on the basis of pleomorphic phenotype. The growth of the T24 bladder cancer cells was systematic (approximately doubled every $24 \mathrm{~h}$ ) on EUV modified surfaces throughout four days of the cell culture. The HCV29 cells also demonstrated good cell adhesion and proliferation. The results indicate laboratory scale compact EUV source as a potential candidate for fabrication of substrates for LOC devices for cancer cell identification.

\section{Acknowledgments}

Authors acknowledge financial support from the EU FP7 Erasmus Mundus Joint Doctorate Programme EXTATIC under framework partnership agreement FPA2012-0033 and from the EU Horizon 2020 research and innovation programme under grant agreement No. 654148 (Laserlab-Europe).

\section{References}

[1] M.-I. Mohammed, M.P.Y. Desmulliez, Lab. Chip 11, 569 (2011).

[2] I.U. Ahad, A. Bartnik, H. Fiedorowicz, J. Kostecki, B. Korczyc, T. Ciach, D. Brabazon, J. Biomed. Mater. Res. A 102, 3298 (2014).

[3] V. Saravia, J.L. Toca-Herrera, Microsc. Res. Tech. 72, 957 (2009).

[4] M. Vázquez, D. Brabazon, F. Shang, J.O. Omamogho, J.D. Glennon, B. Paull, $\operatorname{Tr} A C$ Trends Anal. Chem. 30, 1575 (2011). 
[5] A. Llobera, J. Juvert, A. González-Fernández, B. Ibarlucea, E. Carregal-Romero, S. Büttgenbach, C. Fernández-Sánchez, Light Sci. Appl. 4, e271 (2015).

[6] R.N. Palchesko, L. Zhang, Y. Sun, A.W. Feinberg, PLoS One 7, e51499 (2012).

[7] H. Jin, J. Zhou, X. He, W. Wang, H. Guo, S. Dong, D. Wang, Y. Xu, J. Geng, J.K. Luo, W.I. Milne, Sci. Rep. 3, 2140 (2013).

[8] K. Bagga, R. McCann, M. Wang, A. Stalcup, J. Coll. Interface Sci. 447, 263 (2015).

[9] A. Bartnik, H. Fiedorowicz, R. Jarocki, J. Kostecki, M. Szczurek, P.W. Wachulak, Nucl. Instrum. Methods Phys. Res. A 647, 125 (2011).

[10] I.U. Ahad, B. Budner, B. Korczyc, H. Fiedorowicz, A. Bartnik, J. Kostecki, S. Burdyńska, D. Brabazon, Acta Phys. Pol. A 125, 924 (2014).
[11] I.U. Ahad, H. Fiedorowicz, B. Budner, T.J. Kaldonski, M. Vazquez, A. Bartnik, D. Brabazon, Acta Phys. Pol. A 129, 241 (2016).

[12] A. Bartnik, W. Lisowski, J. Sobczak, P. Wachulak, B. Budner, B. Korczyc, H. Fiedorowicz, Appl. Phys. A 109, 39 (2012).

[13] I.U. Ahad, B. Butruk, M. Ayele, B. Budner, A. Bartnik, H. Fiedorowicz, T. Ciach, D. Brabazon, Nucl. Instrum. Methods Phys. Res. B 364, 98 (2014).

[14] M. Lekka, K. Pogoda, J. Gostek, O. Klymenko, S. Prauzner-Bechcicki, J. Wiltowska-Zuber, J. Jaczewska, J. Lekki, Z. Stachura, Micron 43, 1259 (2012). 